\title{
The Possible Role of Contact Sensitization to Fragrances and Preservatives in Poikiloderma of Civatte
}

\author{
Saranya Khunkhet Penpun Wattanakrai \\ Division of Dermatology, Faculty of Medicine, Ramathibodi Hospital, Mahidol University, \\ Bangkok, Thailand
}

\section{Key Words}

Poikiloderma of Civatte - Kathon CG · Methylchloroisothiazolinone - Methylisothiazolinone . Contact sensitization

\begin{abstract}
Numerous mechanisms have been postulated to play an important role in the pathogenesis of poikiloderma of Civatte (PC), including chronic exposure to ultraviolet radiation, menopause-related hormonal changes, contact hypersensitivity and genetic predisposition. Herein, we report a case of contact sensitization to fragrances and commonly used preservatives, methylchloroisothiazolinone and methylisothiazolinone, also widely known as Kathon CG, in a post-menopausal woman with PC, who denied excessive sun exposure and a family history. After abstaining from the use of her personal care products containing the documented allergens, not only the itching and burning symptoms, but also the cutaneous changes appeared to improve partially. This report underlines the possible influence of contact hypersensitivity in the pathogenesis of PC.

(c) 2014 S. Karger AG, Basel
\end{abstract}

\section{Introduction}

Poikiloderma refers to a skin condition clinically consisting of telangiectasia, cutaneous atrophy and macular hypo- and hyperpigmentation. Poikiloderma of Civatte (PC), an acquired poikiloderma of the face and neck, is a common benign dermatosis often affecting fair-skinned, middle-aged and elderly people. It symmetrically involves the sides and Vshaped area of the neck as well as the lateral parts of the face, with sparing of anatomically 
Khunkhet and Wattanakrai: The Possible Role of Contact Sensitization to Fragrances and Preservatives in Poikiloderma of Civatte

shaded areas [1]. Even though PC has been described for almost a century, its etiopathogenesis is still under investigation. Chronic sun exposure, estrogen depletion associated with menopause, photoallergic as well as allergic contact reactions to perfumes and cosmetics and genetic susceptibility have been proposed as the major culprits [2-4]. In the following report, contact hypersensitivity to fragrances and a mixture of methylchloroisothiazolinone and methylisothiazolinone (MCI/MI), also known as Kathon CG, in a post-menopausal woman with PC will be described.

\section{Case Report}

A 54-year-old Thai woman, who had entered menopause a few years earlier, presented with gradual progression of erythema and hyperpigmentation on the face and neck for 2 years. The patient also complained of moderately burning and itching sensations on the lesional skin. She denied a history of frequent sun exposure and photosensitivity. She had never experienced an allergic reaction to medications, food, perfumes or metals. There were no similar skin conditions running in her family.

Physical examination showed prominent telangiectasias combined with skin atrophy and mottled brown hyperpigmentation on the upper chest, neck, chin, lateral cheeks and periorbital areas (fig. 1a). A skin biopsy, performed on the lesion on the neck, revealed mild epidermal atrophy with effacement of rete ridges and irregularly distributed melanin in the basal layer of the epidermis. Solar elastosis, dilated blood vessels, melanophages and superficial perivascular infiltration of lymphocytes were also noticed in the upper dermis. Based on physical examination and histopathological findings, the diagnosis of PC was established.

Phototesting, patch tests and photopatch tests were performed to evaluate the possible etiology. Phototesting indicated a normal minimal erythema dose to both UVA and UVB. The patient was patch-tested with standard screening series and vehicle-preservative series, and photopatch-tested with Scandinavian standard photopatch series, sunscreen series and NSAID series. Photopatch testing showed negative results while patch testing revealed allergic contact reactions to the preservative $\mathrm{MCI} / \mathrm{MI}$ and several fragrance allergens. In detail, the patient exhibited extremely positive reactions (+++) to fragrance mix I and perfume mix, and strong positive reactions (++) to fragrance mix II and MCI/MI.

Considering the relevance of the positive patch test results, the patient had never encountered typical eczematous changes on the face and neck, but she reported burning and itching sensation after applying some facial creams and sunscreen products. On examination of the patient's cosmetics and toiletries, fragrance, MCI and MI were found in many products she used on a daily basis, such as skin care creams, shampoos, conditioners and soaps. Interestingly, both fragrance and MI allergens were found in the moisturizing cream she regularly applied to her face and neck. Moreover, the repeated open application test with the mentioned moisturizing cream was performed on the patient's forearm and it showed a positive reaction within 12 days.

In terms of management, the patient was advised to practice adequate strategies for sun protection. As the contact sensitization to fragrances and preservatives was suspected to be a causative factor for at least the cutaneous symptoms in this patient, it made the rationale for allergen avoidance and immunomodulatory agents. The patient was also advised to stop using all products containing fragrances and $\mathrm{MCI} / \mathrm{MI}$, and topical 1\% pimecrolimus cream was prescribed for the lesional area. As a result, her itching and burning symptoms significantly decreased within 2 months after start of allergen avoidance. In addition, the cu- 
taneous lesions appeared to improve partially (fig. 1b). However, for cosmetic reasons the patient requested treatment with vascular lasers to reduce the erythema and telangiectasias on the face.

\section{Discussion}

PC, an acquired poikiloderma of the face and neck, was first described by Achille Civatte in 1923 [5]. It is a common chronic dermatosis, particularly in countries where light-skinned people are the majority. Individuals in their fourth to seventh decades are most affected, with a marked female predominance. Clinically, PC manifests as reticulated red to brown patches admixed with telangiectasias, and sometimes cutaneous atrophy, predominantly on the sides and V-shaped area of the neck. The parotid area of the face and the chin are less often involved [1-3]. The symmetrical distribution and sparing of the submental area shaded by the chin are highly characteristic features of the disease [3]. PC tends to have a gradual onset and progressive course. Additionally, up to almost half of patients experience abnormal sensations, described as pruritus, burning or rosacea-like flushing [2, 3]. Because of the variations in clinical appearance, a PC classification was proposed to categorize the disease into erythemato-telangiectatic type, pigmented type and mixed type, which also assisted in determining the most appropriate treatment [3].

The histological findings of PC are characteristic, but not pathognomonic. The most constant features are solar elastosis and dilated blood vessels in the papillary dermis, with the degree of vessel dilatation appearing to depend on the severity of solar elastosis. The main change is thought to be a degenerative process in the dermal connective tissue, leading to dilatation of adjacent blood vessels due to the loss of vascular support. Less predominantly, irregular distribution of melanin in the lower epidermis and presence of melanophages in the upper dermis are frequently observed. Flattened epidermis with effacement of rete ridges, hyperkeratosis and mild perivascular lymphocytic infiltration are also present to a lesser extent. In some cases, vacuolar degeneration of the basal keratinocytes is evidently noticed [6].

To date, the exact etiology and pathogenesis of PC are still obscure. Many causative factors have been hypothesized to lie behind the development of disease, and PC seems not to be influenced by only one factor.

First of all, both the predilection sites for PC and the presence of solar elastosis indicate a pivotal role of cumulative ultraviolet exposure in PC development. However, many PC patients have no history of excessive sun exposure and other sun-exposed areas, such as the forehead and forearms, are not commonly affected. The area susceptibility in PC may be due to (1) the natural photoprotection associated with epidermal thickness, which is thicker in the face and forearm in comparison with the neck area, or (2) inadequate sun protection measures [2]. Broad-brimmed hats are needed to provide maximum protection of the face and neck; nonetheless, a number of people still use just a baseball cap when going outside, leaving the sides of the face and neck exposed to sunlight.

Second, the age and sex distribution of PC patients dictates the possible role of hormonal depletion during menopause. Moreover, $\mathrm{PC}$ also develops in younger women after iatrogenic premature menopause $[3,6]$. As low estrogen levels cause decreased synthesis of collagen and loss of skin elasticity [7], these changes in dermal connective tissue due to estrogen depletion may be responsible for the development of telangiectasia and cutaneous atrophy in PC patients. In addition, genetic factors are suspected to be involved in the pathogenesis as 
Khunkhet and Wattanakrai: The Possible Role of Contact Sensitization to Fragrances and Preservatives in Poikiloderma of Civatte

PC has been reported in families with an autosomal dominant manner, despite sharing no environmental or hormonal factors [4].

Furthermore, the regional distribution of lesions raises the hypothesis that contact dermatitis is another possible explanation for PC. Even though allergic contact dermatitis usually presents with an eczematous lesion, other cutaneous forms, called 'non-eczematous eruptions', have been described to be a result of delayed-type hypersensitivity as well, including a reaction with only pigmentary changes known as pigmented contact dermatitis [8]. Melanin incontinence, a main histopathological feature of pigmented contact dermatitis, is also found in most cases of PC [6]. Thus, these facts may imply that PC probably develops via type IV hypersensitivity similar to pigmented contact dermatitis, or that contact sensitization possibly contributes to the pigment alteration in PC. The preferred sites for PC are areas where perfume is frequently atomized and metal jewelry is commonly used, pushing the possibility that fragrances or metal allergens might be crucial sensitizers. As sparing of anatomically shaded areas is a prominent feature of PC, photocontact sensitization to substances applied to the face and neck may be an additionally suggested mechanism, in combination with the effects of natural photoprotection or inadequate sun protection measures.

With regard to supporting evidence, $\mathrm{MCI} / \mathrm{MI}$ was reported to be a contact sensitizer in a female patient who developed PC in her adolescence, and skin lesions considerably improved after allergen avoidance [9]. Later, a case-control study evaluating the role of contact dermatitis in 32 PC patients documented that a higher rate of positive patch testing to fragrances was found in PC patients compared to age- and sex-matched controls who had a suspected contact dermatitis of the face and/or neck, accounting for 25.0 and $9.27 \%$, respectively $(\mathrm{p}<0.05)$. There was no statistically significant difference of positive patch testing to nickel between these two groups [2]. Interestingly, positive patch testing to $\mathrm{MCI} / \mathrm{MI}$ was also shown in one PC patient [2]. On the other hand, there are only few data supporting the postulation of PC being a photoallergic contact disease. Photocontact sensitization to 6-methylcoumarin, a fragrance allergen, was reported in a post-menopausal woman with PC who had no history of chronic sun exposure. This fragrance allergen was also found in the patient's regularly used perfume [10]. However, in the largest study of PC mentioned above, the results of photopatch testing were negative in all tested PC patients [2].

Our patient is a case of symptomatic PC in a post-menopausal woman without an obvious history of sun exposure or family history. Her complaint of burning/itching symptoms appeared to be notable and the skin changes appeared to be beyond the usual locations. Subsequently, contact hypersensitivity to fragrances and MCI/MI was demonstrated by showing strong positive patch test reactions whereas typical eczematous lesions had never occurred on the face and neck areas. The patient denied the use of perfume or cologne; however, fragrance as well as MCI/MI were listed as ingredients in many of her leave-on and rinse-off personal care products. In fact, both allergens were found in the moisturizing cream she regularly applied only to her face and neck, and it was suspected to be the main culprit causing skin changes on these areas. For PC, the patient was advised to avoid sun exposure and to regularly use sunscreen. Regarding her symptoms and patch test results, we recommended her to stop using products containing the documented allergens and to apply topical pimecrolimus cream to the atrophic telangiectatic skin lesions. Consequently, the itching and burning sensations considerably decreased and the erythematous change partially improved before the patient elected to initiate additional laser treatment on the face to improve her clinical appearance.

We propose that contact hypersensitivity to fragrances and MCI/MI might be a contributing etiology of PC in this patient, in combination with the impact of estrogen decline in her menopause and cumulative sun exposure. A trial of allergen and sun avoidance should be 
recommended to patients as it may have some effect on disease improvement. Apart from fragrance allergens, which have been reported as causative agents in PC [2], MCI/MI may probably be another important sensitizer which should be under the radar of investigation. The reported rate of contact hypersensitivity to MCI/MI has constantly risen since 2005 when MI was permitted to be used alone as a preservative at a much higher maximum concentration compared to the maximum allowed concentration of MI when it is combinedly used with $\mathrm{MCI}$ in MCI/MI [11]. As a consequence, MCI/MI-induced PC may possibly be seen to occur more often in parallel with the increased prevalence of MCI/MI sensitization.

In conclusion, we suggest that patients with $\mathrm{PC}$, particularly those experiencing pruritus or burning sensations with a history of topical applications to their affected areas, should undergo patch and photopatch testing to evaluate the possible causes, as the avoidance of positively identified allergens may benefit the improvement of PC.

\section{Disclosure Statement}

The authors declare that there is no conflict of interests regarding the publication of this paper.

\section{References}

$\checkmark 1$ Nofal A, Salah E: Acquired poikiloderma: proposed classification and diagnostic approach. J Am Acad Dermatol 2013;69:e129-e140.

-2 Katoulis AC, Stavrianeas NG, Katsarou A, Antoniou C, Georgala S, Rigopoulos D, Koumantaki E, Avgerinou G, Katsambas AD: Evaluation of the role of contact sensitization and photosensitivity in the pathogenesis of poikiloderma of Civatte. Br J Dermatol 2002;147:493-497.

-3 Katoulis AC, Stavrianeas NG, Georgala S, Bozi E, Kalogeromitros D, Koumantaki E, Katsambas AD: Poikiloderma of Civatte: a clinical and epidemiological study. J Eur Acad Dermatol Venereol 2005;19: 444-448.

-4 Katoulis AC, Stavrianeas NG, Georgala S, Katsarou-Katsari A, Koumantaki-Mathioudaki E, Antoniou C, Stratigos JD: Familial cases of poikiloderma of Civatte: genetic implications in its pathogenesis? Clin Exp Dermatol 1999;24:385-387.

5 Civatte A: Poïkilodermie réticulée pigmentaire du visage et du cou. Ann Dermatol Syphilol 1923;6:605-620.

6 Katoulis AC, Stavrianeas NG, Panayiotides JG, Bozi E, Vamvasakis E, Kalogeromitros D, Georgala S: Poikiloderma of Civatte: a histopathological and ultrastructural study. Dermatology 2007;214:177-182.

7 Shu YY, Maibach HI: Estrogen and skin: therapeutic options. Am J Clin Dermatol 2011;12:297-311.

8 Shenoi SD, Rao R: Pigmented contact dermatitis. Indian J Dermatol Venereol Leprol 2007;73:285-287.

-9 Sahoo B, Kumar B: Role of methylchloroisothiazolinone/methylisothiazolinone (Kathon CG) in poikiloderma of Civatte. Contact Dermatitis 2001;44:249.

10 Vachiramon V, Wattanakrai P: Photoallergic contact sensitization to 6-methylcoumarin in poikiloderma of Civatte. Dermatitis 2005;16:136-138.

11 Castanedo-Tardana MP, Zug KA: Methylisothiazolinone. Dermatitis 2013;24:2-6. 


\section{Case Reports in Dermatology}

\begin{tabular}{l|l}
\hline Case Rep Dermatol 2014;6:258-263 \\
\hline DOI: 10.1159/000369489 & $\begin{array}{l}\text { C 2014 S. Karger AG, Basel } \\
\text { www.karger.com/cde }\end{array}$ \\
\hline
\end{tabular}

Khunkhet and Wattanakrai: The Possible Role of Contact Sensitization to Fragrances and Preservatives in Poikiloderma of Civatte
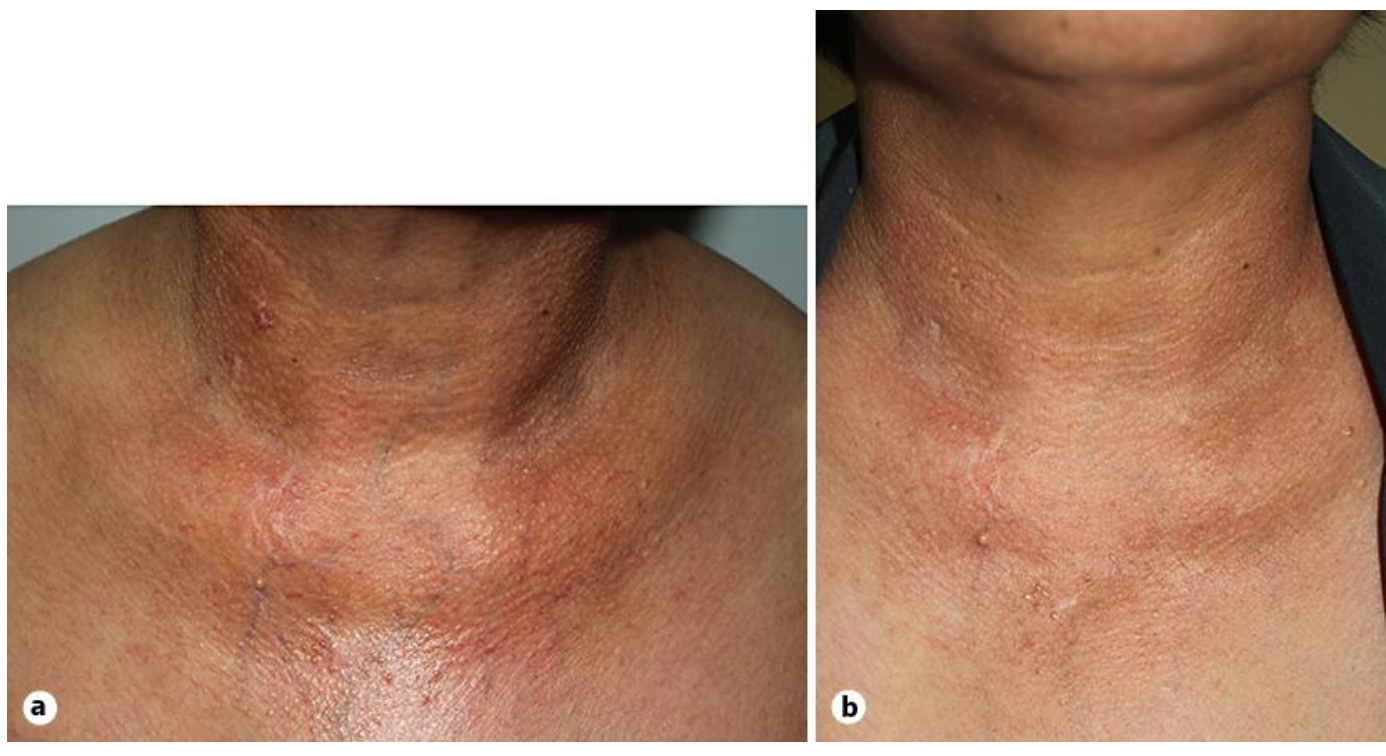

Fig. 1. Prominent telangiectasias admixed with cutaneous atrophy and hyperpigmentation on the upper chest and the neck. Cutaneous changes appeared to improve after allergen and sun avoidance. a Before treatment. b After treatment. 